

\title{
Informing patients of relevant health information and innovations: patient recall or annual physical?
}

\author{
Authors: I. Mastroleo, F. Holzer, R. Mertelsmann
}

\begin{abstract}
Recently the NEJM published an interesting debate on the value of the annual physical (also known as "periodic health examination") (Goroll 2015). "Today, because of the rapid growth of medical knowledge and the widening application of technology to medicine, there is a particular need for a continuing authoritative review of, standards for both preventive and therapeutic strategies. Some of these strategies are subsumed under the general category of the periodic health examination." (Canadian Task Force on the Periodic Health Examination 1979). Although the current debate of the annual physical has narrowly focused on preventive strategies in healthy individuals and building patient-physicians relationship, recent breakthrough clinical innovation in many fields, especially in genomic medicine, widens the need of review to new effective therapy and diagnostics. This is the case because genomic medicine indicates new therapeutic treatments and targets, and the costs of sequencing have been decreasing over time.
\end{abstract}

\section{Article}

Recently the NEJM published an interesting debate on the value of the annual physical (also known as "periodic health examination") (Goroll 2015). Since the late 1970s, the "annual physical" has been recognized as one of the most important strategies of a broader need of continuing review of medical innovations and interventions given the rapid growth of medical knowledge and technological applications:

"Today, because of the rapid growth of medical knowledge and the widening application of technology to medicine, there is a particular need for a continuing authoritative review of, standards for both preventive and therapeutic strategies. Some of these strategies are subsumed under the general category of the periodic health examination." (Canadian Task Force on the Periodic Health Examination 1979)

Although the current debate of the annual physical has narrowly focused on preventive strategies in healthy individuals and building patient-physicians relationship, recent breakthrough clinical innovation in many fields, especially in genomic medicine, widens the need of review to new effective therapy and diagnostics. This is the case because 
genomic medicine indicates new therapeutic treatments and targets, and the costs of sequencing have been decreasing over time.

In turn, this makes us wonder about the impact of new and continuously growing clinical and genomic knowledge both in preventive and therapeutic strategies. It also brings to light the broader need of a continuing review of preventive and therapeutic and diagnostic standards and the corresponding duty of informing patients of relevant health innovations for their conditions.

Although this applies only to persons with a genomic sequence done, new genomic as well as other medical knowledge can influence therapeutic, diagnostic and preventive health decisions. Even if a new pathological variant might not be treated or prevented, patients carrying such a variable may want to take the appropriate measures to prevent passing it on to future generations. With the gradually increase of genomic sequencing both in clinical research and clinical practice, this concerns will only grow bigger.

As Mehrotra \& Prochazka (2015) argue "[...] new criteria for meaningful use of electronic health records emphasize active surveillance to ensure that preventive care services are up to date". These criteria should also be applicable to ensure that therapeutic care services are up to date too. And it might include stored individual genomic sequences in both research and clinical database.

However, the problem is that without an effective means to feedback of relevant new information to patients, "updating" preventive and therapeutic care remains at best, a good aspiration and at worst, a source of concern if it is not properly implemented. So the fundamental question is the following, have we reach the point that continuous review and feedback of relevant new medical information to patients is ethically obligatory? If ethical "must" implies practical "can", we think that the answer is negative in a wide range of cases.

However, there are some important considerations to make. While new communication technologies have increased our capabilities to reach general patients population at low cost, lack or shortage in genetic counseling services might pose a yet unsurmountable barrier in many parts of the world. However, where counseling services are available, the moral force of the requirement of informing patients of relevant health innovations is strong. Furthermore, it grows even stronger even when genetic counselling services are minimally available, if the target population for feedback is carefully selected making practicality concerns shrink or fade. So in principle, there's no ethical duty for active recall 
general patients population to inform relevant health innovations, but it might be a limited ethical duty for active recall of special patient populations.

However, current practicality concerns do not get physicians and society off the ethical hook of the ethical requirement of informing general patients' population of relevant health innovations completely. What we argue here is that the annual physical is currently the most appropriate mechanism for physicians and health systems to operationalize the ethical requirement of informing patients of relevant health innovations.

Although this is not a definitive argument in favor of the annual physical, we believe it brings a central missing aspect to this stablished mechanism of physician-patient relationship. By "skipping the annual physical" as Ezequiel Emanuel (2015) recommends, patients also skip one of the few possibilities to engage in relevant and meaningful feedback on relevant health information and innovations given by their physicians. We do recognize that the process of the annual physical should be revised, eliminating unnecessary procedures and costs, and more data should be produce to show if it is beneficial, as part of the continuous scientific evaluation of accepted practice. However, it should include an in-depth review of those advances in medicine and genomics that are relevant to the specific patient. A crucial aspect of such a communication process is necessary for a truly "a close, trusting relationship with the doctor" (Goroll 2015).

\section{References}

Canadian Task Force on the Periodic Health Examination. (1979). The periodic health examination. Canadian Medical Association Journal, 121(9), 1193-1254.

Emanuel, E. J. (2017, December 21). Skip Your Annual Physical. The New York Times. Retrieved from https://www.nytimes.com/2015/01/09/opinion/skip-your-annualphysical.html

Goroll, A. H. (2015). Toward Trusting Therapeutic Relationships - In Favor of the Annual Physical. New England Journal of Medicine, 373(16), 1487-1489. http://doi.org/10.1056/NEJMp1508270

Mehrotra, A., \& Prochazka, A. (2015). Improving Value in Health Care - Against the Annual Physical. New England Journal of Medicine, 373(16), 1485-1487. http://doi.org/10.1056/NEJMp1507485 